\title{
Bias against disconfirmatory evidence in the 'at-risk mental state' and during psychosis
}

\author{
Sarah Eisenacher ${ }^{\mathrm{a}, *}$, Franziska Rausch ${ }^{\mathrm{a}}$, Daniela Mier ${ }^{\mathrm{b}}$, Sabrina Fenske $^{\mathrm{b}}$, \\ Ruth Veckenstedt ${ }^{c}$, Susanne Englisch ${ }^{\mathrm{a}}$, Anna Becker ${ }^{\mathrm{a}}$, Christina Andreou ${ }^{\mathrm{c}}$, Steffen Moritz ${ }^{\mathrm{c}}$, \\ Andreas Meyer-Lindenberg ${ }^{a}$, Peter Kirsch ${ }^{\mathrm{b}}$, Mathias Zink ${ }^{\mathrm{a}}$ \\ a Department of Psychiatry and Psychotherapy, Central Institute of Mental Health, University of Heidelberg/Medical Faculty Mannheim, J5, 68159 Mannheim, \\ Germany \\ b Department of Clinical Psychology, Central Institute of Mental Health, University of Heidelberg/Medical Faculty Mannheim, J5, 68159 Mannheim, Germany

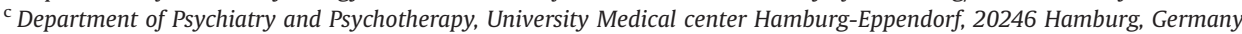

\begin{abstract}
A B S T R A C T
Prior studies have confirmed a bias against disconfirmatory evidence (BADE) in schizophrenia which has been associated with delusions. However, its role in the pathogenesis of psychosis is yet unclear. The objective was to investigate BADE for the first time in subjects with an at-risk-mental-state for psychosis (ARMS), patients with a first episode of psychosis without antipsychotic treatment (FEP) and healthy controls (HC). A standard BADE test presenting written scenarios was employed. In addition, psychometric rating scales and a neuropsychological test battery were applied. A three-staged image was revealed. FEP-patients showed a significant BADE compared to the other groups. The performance of ARMS-patients lay in between HC and FEP-patients. A trend towards significance became evident for a bias against confirmatory evidence (BACE) in FEP-patients. Results were not attributable to antipsychotic or other medication or depressive symptoms. Correlations with delusions reached medium effect sizes but failed significance after Bonferroni-corrections. These results provide evidence for aberrations in evidence integration in the pathogenesis of psychosis and contribute to our knowledge of metacognitive functioning which can be used for (meta-)cognitive intervention in psychosis.
\end{abstract}

\section{Introduction}

The investigation of cognitive aberrations in schizophrenia has gained increasing attention because they represent potential cognitive mechanisms of the development and maintenance of delusions (Garety et al., 2001). So-called metacognitive biases, namely impairments in controlling and monitoring one's own cognitive processes, include for example the jumping to conclusion bias (i.e. a bias in data-gathering leading to an early decision making on the basis of little information) (Esslinger et al., 2013; Fine et al., 2007; Jolley et al., 2014; Rausch et al., 2014, 2015) or a metamemory bias (i.e. a limited differentiation in confidence between correct and false memories) (Eifler et al., 2014b; Eisenacher et al., 2015). Furthermore, many recent studies have concentrated on belief inflexibility (So et al., 2012), such as a bias in evidence

\footnotetext{
* Corresponding author at: Central Institute of Mental Health, Department of Psychiatry and Psychotherapy Medical Faculty Mannheim/Heidelberg University, P.O. Box 1221 20, D-68072 Mannheim, Germany.

E-mail address: sarah.eisenacher@zi-mannheim.de (S. Eisenacher).
}

integration (e.g. Speechley et al., 2012) made of a bias towards both disconfirmatory evidence (BADE) and confirmatory evidence (BACE) (Eifler et al., 2014a; Woodward et al., 2008). The BADE describes a decreased ability to adjust one's beliefs according to information which does not support one's hypothesis. The BACE, on the other hand, describes a difficulty to integrate information which supports the true answer. The ability to integrate new information into the reasoning process is important in order to adapt one's beliefs according to new knowledge. Not changing one's beliefs in the presence of disconfirming information and holding them with strong conviction though they are proved to be false is a hallmark of delusions as defined by Karl Jaspers (Jablensky, 2013).

Woodward et al. (2006b) were the first to introduce a paradigm to measure BADE and BACE. Pictures were disambiguated over time by additional confirmatory and disconfirmatory information. Later the paradigm was refined and written scenarios were presented (Buchy et al., 2007). The change of plausibility ratings for lure or true interpretations over the course of information growth indicated BADE or BACE, respectively. Strength of these paradigms 
is their usage of delusion neutral material precluding tautological reasoning. The authors reported findings of BADE in delusionalcompared to non-delusional patients and in all patients compared to healthy controls (Woodward et al., 2006b). In subsequent studies, a BADE in schizophrenia patients was often replicated in comparison to healthy (e.g. Riccaboni et al., 2012; Veckenstedt et al., 2011; Woodward et al., 2006a, 2008) and psychiatric control groups (Moritz and Woodward, 2006; Veckenstedt et al., 2011). Summarized, these findings propose that patients with schizophrenia are less able to integrate into their reasoning process new information that could call their current beliefs into question. In contrast, findings about a bias against confirmatory evidence (BACE) are mixed (Eifler et al., 2014a; Riccaboni et al., 2012; Veckenstedt et al., 2011). In addition, a third aberration, namely a liberal acceptance tendency, was found in several studies, demonstrating that patients with schizophrenia often rated implausible interpretations with higher plausibility than controls (Eifler et al., 2014a; Moritz and Woodward, 2004; Woodward et al.,. 2006b).

In a prior theoretical paper it was outlined that the integration of disconfirmatory evidence might be a risk factor for the maintenance of delusions (Garety et al., 2001). Supporting this theory, multiple experimental studies also revealed that a BADE was specifically associated with delusion severity (Riccaboni et al., 2012; Sanford et al., 2014; Speechley et al., 2012; So et al., 2012; Woodward et al., 2006b). Some other studies which did not observe associations between BADE and delusions discussed BADE as a potential trait-factor for delusions rather than a state-dependent bias (Moritz and Woodward, 2006; Veckenstedt et al., 2011). As most studies have usually included chronic patients with antipsychotic medication, the question emerges whether the findings of BADE might be confounded by this factor. So et al. (2010) proposed that belief flexibility mediates the effect of antipsychotic medication on symptoms implying that a symptom reduction by antipsychotics is reached by a reduction of belief inflexibility. Similarly, improved evidence integration ability under single-dose haloperidol compared to single-dose L-dopa in healthy controls suggests an association between both variables (Andreou et al., 2015). To exclude antipsychotic effects as confounds on evidence integration ability it is important to recruit patients prior to this medication.

Based on this set of findings, the role of evidence integration in the dynamic development of psychosis seems to be important. Prior literature has shown that healthy participants displayed a BADE in correlation with higher delusion ideation (Buchy et al. 2007; Orenes et al., 2012; Woodward et al., 2007; Zawadzki et al., 2012). However, no study up to date has explored this topic in risk constellations for psychosis, meaning those patients in an "at-risk mental state" (ARMS) for psychosis either presenting with cognitive basic symptoms and/or attenuated psychotic symptoms and/ or brief limited intermittent psychotic symptoms (Fusar-Poli et al., 2012; Fusar-Poli et al., 2013; Schultze-Lutter et al., 2010, 2012). Such an investigation could enhance our knowledge about potential associations with early psychosis-prone symptoms. By differentiating between those symptoms, it is also possible to classify and examine subgroups of ARMS-patients. Some evidence regarding other metacognitive aberrations suggests a three-staged image of performance with ARMS-patients being less impaired than patients with a first episode of psychosis (FEP) but more than healthy controls (Eisenacher et al., 2015). Within this scope, it is also worth investigating possible associations between evidence integration and neurocognitive performance. Previous studies have demonstrated inconsistent results, reporting either no associations (Moritz et al., 2010; Woodward et al., 2007), associations with executive functioning, verbal learning, and theory of mind (Riccaboni et al., 2012), or with executive functioning, processing speed, working memory and vigilance (Eifler et al., 2014a) in patients with schizophrenia. Whether associations can be found in risk constellations for psychosis is open to question.

To our knowledge, the present study is the first focusing on evidence integration in ARMS-patients. Our main aim was to investigate patterns of evidence integration between ARMS-patients, antipsychotic-free FEP-patients and healthy controls. We expected three levels of performance with increasing impairment from healthy controls over ARMS-patients to FEP-patients. Furthermore, we hypothesized that BADE would be associated with measures of delusions and neurocognitive performance in the patient groups. In a tertiary analysis, we compared subgroups of ARMS-patients regarding BADE and BACE. We hypothesized to find less evidence integration in groups displaying higher delusional conviction.

\section{Methods}

\subsection{Participants}

The present study was conducted as part of a larger study about metacognitive and neurocognitive deficits in schizophrenia and ARMS-patients ("Metacognitive deficits in patients with at-risk mental states for schizophrenia and their interaction with psychopathology, cognitive dysfunction and functional imaging"). The study was approved by the local ethical board of the Medical Faculty Mannheim of the Ruprecht-Karls-University Heidelberg (Germany; accession number: 2009-296N-MA). All participants were carefully informed about aims and procedures of the study and provided their written consent. We investigated evidence integrationperformance in 44 ARMS-patients, 28 FEP-patients, and 38 healthy controls. ARMS-patients were recruited via the Early Recognition Outpatient Unit of the Central Institute of Mental Health in Mannheim, Germany. They were allocated to an ARMS according to a diagnostic interview with the Early Recognition Inventory based on IRAOS (ERIraos) (Häfner et al., 2012; Rausch et al., 2013) defined by the transgression of the cut-off (sum score $\geq 30$ ) and/or the presence of at least two cognitive basic symptoms and/or at least one attenuated psychotic symptom and/ or at least one brief limited intermittent psychotic symptom within the last 12 months. The ERIraos has been demonstrated to be equally sensitive in the detection of attenuated psychotic symptoms and brief limited intermittent psychotic symptoms compared to the Comprehensive Assessment of At-Risk Mental State (CAARMS; Yung et al., 2005). By the inclusion of cognitive basic symptoms, it furthermore allows for a higher sensitivity in the assessment of the whole spectrum of ARMS-symptoms and thereby reduces false-negative attributions (Rausch et al., 2013). In addition to the investigation of the total ARMS-group, subgroups of ARMS-patients were identified by differentiating between groups of symptoms. One group $(n=13)$ consisted of patients who presented only with cognitive basic symptoms, the second group $(n=16)$ consisted of patients with at least one attenuated psychotic symptom, and a last group $(\mathrm{n}=15)$ consisted of patients with at least one brief limited intermittent psychotic symptom. FEP-patients were recruited via the Early Recognition Outpatient Unit or during their inpatient treatment in the Central Institute of Mental Health and fulfilled diagnostic criteria of a first episode of psychosis according to the Diagnostic and Statistical Manual IV (Saß et al., 2000). All groups fulfilled predefined inclusion criteria: 1) age between 18 and 40 years; 2) ability to provide informed consent; 3) sufficient German language skills. Exclusion criteria for patients were as follows: 1) antipsychotic medication for more than four weeks in total and during the last four weeks prior to testing; 2) substance dependence excluding nicotine; 3) other disorders of the central nervous system requiring treatment. 
Table 1

Sociodemographic data and clinical characteristics.

\begin{tabular}{|c|c|c|c|c|c|}
\hline & \multirow[t]{2}{*}{ HC $(n=38)$} & \multirow[t]{2}{*}{ ARMS $(n=44)$} & \multirow[t]{2}{*}{ FEP $(n=28)$} & \multicolumn{2}{|c|}{ Group comparison } \\
\hline & & & & Test & $\mathrm{p}$ \\
\hline \multicolumn{6}{|l|}{ Sociodemography } \\
\hline Age (in years) & $25.08(6.61)$ & $23.45(4.87)(10.72)$ & $26.14(4.93)$ & $F(2,107)=2.14$ & .12 \\
\hline Sex (male/female) & $25 / 13$ & $30 / 14$ & $19 / 9$ & $\chi^{2}(2)=.59$ & .97 \\
\hline Years of education & $11.42(1.52)$ & $11.36(1.57)$ & $10.64(1.79)$ & $F(2,107)=2.26$ & .11 \\
\hline Premorbid intelligence (MWT-B) & $95.76(10.69)$ & $96.64(10.48)$ & $91.46(7.81)$ & $F(2,107)=2.47$ & .09 \\
\hline \multicolumn{6}{|l|}{ Clinical characteristics } \\
\hline ERIraos sum score & - & $43.16(14.96)$ & - & - & - \\
\hline PANSS positive & - & $14.52(4.75)$ & $23.21(6.77)$ & $t(70)=-6.40$ & $<.001$ \\
\hline PANSS negative & - & $17.93(6.87)$ & $21.07(9.14)$ & $t(70)=-1.66$ & .101 \\
\hline PSYRATS conviction & - & $1.45(1.58)$ & $3.06(1.17)$ & $t(70)=-4.56$ & $<.001$ \\
\hline SANS & - & $31.52(20.65)$ & $40.82(23.56)$ & $t(70)=-1.76$ & .08 \\
\hline CDSS & - & $7.05(4.77)$ & $5.18(5.14)$ & $t(70)=1.57$ & .12 \\
\hline GAF & - & $48.20(11.25)$ & 31.46 (6.99) & $t(70)=7.05$ & $<.001$ \\
\hline PSP & - & $58.07(15.82)$ & $46.43(12.83)$ & $t(70)=3.27$ & .002 \\
\hline CGI-severity & - & $4.18(.58)$ & $5.29(.66)$ & $t(70)=-7.46$ & $<.001$ \\
\hline
\end{tabular}

Note: Data are presented as mean (standard deviation).

Abbreviations: ARMS = at-risk mental state, ERIraos=Early Recognition Inventory based on IRAOS, FEP=first episode of psychosis, GAF $=$ Global Assessment of Functioning, $\mathrm{GPP}=$ General Psychopathology, $\mathrm{HC}=$ healthy controls, IRAOS=Interview for the Retrospective Assessment of the Onset of Schizophrenia and Other Psychoses, PANSS= Positive and Negative Syndrome Scale, PSP = Personal and Social Performance Scale, PSYRATS=Psychotic Symptom Rating Scales.

Healthy control participants were carefully characterized regarding family history of schizophrenia and bipolar disorder in firstdegree relatives, previous or current psychiatric disorders using the Mini-International Neuropsychiatric Interview (Sheehan et al., 1998), former or present psychopharmacological treatment and abuse of illegal substances within four weeks prior to the investigation and were excluded if they fulfilled any of these criteria.

All groups were matched regarding gender, age, levels of education and premorbid verbal intelligence (Table 1). Due to reasons of feasibility we allowed stable treatment with antidepressants or benzodiazepines. Eleven ARMS- and four FEP-patients were treated with antidepressant agents (ARMS: citalopram $n=2$, duloxetine $\mathrm{n}=1$, mirtazapine $\mathrm{n}=3$, paroxetine $\mathrm{n}=1$, sertraline $\mathrm{n}=2$, trimipramine $n=2$; FEP: amitriptylin $n=1$, citalopram $n=1$, trimipramine $n=1$, venlafaxine and mirtazapine $n=1$ ) or received low doses of lorazepam or diazepam (ARMS: $\mathrm{n}=4$; mean diazepam-equivalent according to Ashton (2002): $8.75 \pm 1.44$; FEP: $\mathrm{n}=17$, mean diazepam-equivalent: $15.29 \pm 11.07$ ).

\subsection{Procedures}

Current ARMS-symptoms, psychosis-symptoms and general psychopathology were assessed by trained and certified raters (FR, SEi) using ERIraos, the Positive and Negative Syndrome Scale (PANSS; Kay et al., 1987) and the delusion part of the Psychotic Symptoms Rating Scales (PSYRATS; Haddock et al., 1999). Using the five-factor model, introduced by van der Gaag et al. (2006), we evaluated subscales of positive and negative symptoms, excitement, emotional distress and disorganization within the PANSS. Furthermore, negative and depressive symptoms were evaluated with the Scale for the Assessment of Negative Symptoms (Andreasen 1989) and the Calgary Depression Scale for Schizophrenia (Addington et al., 1993). We rated illness severity using the Clinical Global Impression Scale (National Institute of Mental Health, 1970) and social and global functioning using the Global Assessment of Functioning (Jones et al., 1995) and the Personal and Social Performance Scale (Patrick et al., 2009).

Neurocognitive functioning was assessed using the MATRICS (Measurement and Treatment Research to Improve Cognition in Schizophrenia) consensus cognitive battery for schizophrenia (Nuechterlein et al., 2008). Following neurocognitive domains are covered by the battery: processing speed, attention/vigilance, verbal and visual learning, working memory, planning, social cognition. Additionally, we tested for set shifting and maintenance using the Wisconsin Card Sorting Test (Heaton et al., 1993) and for set maintenance and alternate attention using the Trail Making Test, version B (Reitan and Wolfson, 1995). Premorbid verbal intelligence was estimated by means of the German Multiple Choice Word Test, version B (Lehrl, 2005).

\subsubsection{The BADE task}

Evidence integration was evaluated by a German version of the BADE task (Veckenstedt et al., 2011; Woodward et al., 2007). Details were published earlier (Eifler et al., 2014a). Shortly summarized, the task consists of 12 written scenarios which are described by three progressively disambiguating sentences each. Four types of interpretation (true, neutral lure, emotional lure, and absurd) have to be rated for plausibility after each sentence. Lure interpretations lose plausibility after the first sentence, whereas the true interpretation gains plausibility. The absurd interpretation is implausible across all sentences. Four control scenarios, in which true interpretations are most plausible from the first sentence onwards, are inserted to mask the pattern of the task but excluded from statistical analyses. An example of the task is given in Appendix A. Each participant was individually tested in a quiet room. The task started with one practice trial. Trial order and position of the four interpretations were presented randomized across participants. Moreover, with regard to follow-up measurements, each participant was randomized to one of three equivalent versions of the task.

\subsection{Statistics}

Data was analyzed using the Statistical Package for Social Sciences (SPSS version 20.0, Chicago, IL, US). We tested for non-normal distributions of parameters using histograms and the Kolmogorov-Smirnov-Test. Sociodemographic data was analyzed using t-tests and univariate analyses of variance. Neuropsychological functioning was analyzed using multivariate analyses of variance. In cases of missing data $(2 \times$ TMT-B, $1 \times$ visual learning, $3 \times$ attention/vigilance, $2 \times$ social cognition) scores were casewise excluded.

In a primary analysis, we concentrated on group differences of evidence integration between ARMS-patients, FEP-patients and 
controls. To explore overall differences in evidence integration patterns in the BADE task, we conducted a $3 \times 3 \times 3$ mixed-model analysis of variance with interpretation type (true answers, lures, absurd answers) and sentence (one, two, three) as independent variables and the group affiliation (healthy controls, ARMS, FEP) as between-group factor. Mean plausibility ratings served as dependent variables. Afterwards, we computed the two types of evidence integration indexes in accordance with previous studies (Veckenstedt et al., 2011; Woodward et al., 2008): 1) BADE was calculated by subtracting the plausibility rating for lure interpretations (mean of neutral and emotional lures) after sentence three off sentence one, 2) BACE was determined by the absolute value of the subtraction of the plausibility rating after sentence three from the rating after sentence one for true interpretations. Higher change scores indicated higher flexibility in evidence integration. These change scores were compared using univariate analyses of covariance (ANCOVA), followed by Bonferroni corrected pairwise comparisons. We included the initial rating after sentence 1 as a covariate to control for potential impacts of initial beliefs on changes in the beliefs. Furthermore, liberal acceptance was analyzed by comparing mean ratings of all absurd items between groups according to Woodward et al. (2006b).

In a secondary analysis, we explored correlations of BADE- and BACE-indexes with psychopathological variables and neuropsychological measures regarding ARMS-patients and FEP-patients separately as well as regarding an "all-patients-" group. We used Pearson's correlation coefficient if data was normally distributed or Spearman rank correlations if prerequisites were not fulfilled and the data could not be improved by standard transformation methods. Bonferroni correction was applied to control for multiple comparisons within all correlational analyses.

In a tertiary analysis, subgroups of ARMS-patients were compared regarding BADE- and BACE-indexes. Univariate analyses of covariance with the initial rating after sentence 1 as covariate were applied.

\section{Results}

\subsection{Sociodemographic data}

FEP-patients showed significantly more positive symptoms as well as a higher social and functional impairment and more illness severity compared to the ARMS-group (Table 1). Regarding neuropsychological performance, results showed significant differences between the control group and FEP-patients in all domains. Performance of ARMS-patients lay intermediate between the other two groups in all domains (Table 2).

\subsection{Primary analysis}

Baseline data of BADE were as follows (means (standard deviations)): healthy controls 3.39 (1.71), ARMS-patients 2.98 (1.71), FEP-patients 2.44 (2.65). BACE data were as follows: healthy controls 5.97 (2.08), ARMS-patients 5.88 (2.03), FEP-patients 4.81 (2.57). Plausibility ratings after each sentence can be found in Table 3.

The mixed-model-analysis revealed a main effect of sentence $(F$ $\left.(1.22,130.88)=35.17, p<.001, \eta_{p}^{2}=.25\right)$ indicating that plausibility ratings increased from sentence one to sentence three. A main effect of interpretation type was revealed $(F(1.82,195.22)=523.76$, $p<.001, \eta_{p}^{2}=.83$ ) indicating that true answers were rated with the highest, lures with intermediate and absurd answers with the lowest plausibility ratings. The main effect of group did not reach significance $\left(F(2,107)=1.04, p=.36, \eta_{p}^{2}=.02\right)$. Further, there was a two-way interaction between sentence and interpretation type ( $F$ $\left.(1.9,203.48)=621.49, p<.001, \eta_{p}^{2}=.85\right)$, which underscored the validity of the task: participants increased their plausibility ratings for true interpretations with consecutive sentences while they decreased their ratings for lure interpretations. The three-way interaction group $\times$ sentence $\times$ interpretation type also reached significance at a medium effect size $(F(3.8,203.48)=3.32, p=.01$, $\left.\eta_{p}^{2}=.06\right)$. Post-hoc analyses of variance using Pillai's trace revealed a group difference for lure interpretations $(F(6,212)=2.25, p=.04$, $\eta_{p}^{2}=.06$ ) which could be attributed to significantly higher plausibility ratings after sentence three of FEP-patients compared to controls $(p=.003,95 \% \mathrm{CI}[.37,1.68])$ and to ARMS-patients ( $p=.01$, $95 \% \mathrm{CI}[-1.45,-.78])$. Liberal acceptance differed between groups $\left(F(2,107)=4.14, p=.02, \eta_{p}^{2}=.07\right)$. Post-hoc analyses revealed a difference between FEP- and ARMS-patients ( $p=.02$, 96\% CI [.79, $1.06])$.

In a next step, the ability to integrate disconfirmatory and confirmatory evidence was analyzed. Group differences of change scores for lure interpretations reached significance $(F(2,106)=$ 5.19, $p=.007, \quad \eta_{p}^{2}=.09$ ). Pairwise comparisons adjusted by

Table 2

Neuropsychology.

\begin{tabular}{|c|c|c|c|c|c|c|c|c|}
\hline & \multirow[t]{2}{*}{ HC $(n=38)$} & \multirow[t]{2}{*}{ ARMS $(n=44)$} & \multirow[t]{2}{*}{ FEP $(n=28)$} & \multicolumn{2}{|c|}{ Group comparison } & \multirow[t]{2}{*}{ HC vs. ARMS } & \multirow[t]{2}{*}{ HC vs. FEP } & \multirow[t]{2}{*}{ ARMS vs. FEP } \\
\hline & & & & Test & $\mathbf{p}$ & & & \\
\hline \multicolumn{9}{|l|}{ MATRICS MCCB } \\
\hline Speed of processing & 51.37 (10.69) & $44.79(8.72)$ & $37.29(12.24)$ & $F(2,107)=17.13$ & $<.001$ & .011 & $<.001$ & .004 \\
\hline Attention/ vigilance & $42.92(8.83)$ & $39.79(9.72)$ & $36.21(12.27)$ & $F(2,104)=4.33$ & .016 & .509 & .012 & .236 \\
\hline Working memory & $54.58(10.31)$ & $49.86(9.30)$ & $44.88(10.42)$ & $F(2,107)=9.12$ & $<.001$ & .091 & $<.001$ & .056 \\
\hline Verbal WM & $50.55(9.78)$ & $48.64(8.21)$ & 43.29 (11.17) & $F(2,107)=4.84$ & .010 & 1.0 & .009 & .068 \\
\hline Visuospatial WM & $56.84(10.68)$ & $50.75(10.15)$ & $46.71(11.14)$ & $F(2,107)=7.75$ & .001 & .032 & .001 & .354 \\
\hline Verbal learning & $57.58(11.91)$ & $50.30(10.95)$ & 44.75 (11.09) & $F(2,107)=12.24$ & $<.001$ & .011 & $<.001$ & .067 \\
\hline Visual learning & $50.34(7.89)$ & 47.56 (11.39) & 42.79 (11.99) & $F(2,106)=5.33$ & .006 & .717 & .005 & .082 \\
\hline Reasoning/planning & $51.95(7.27)$ & $46.42(8.99)$ & $39.54(8.49)$ & $F(2,107)=18.03$ & $<.001$ & .012 & $<.001$ & .002 \\
\hline Social cognition & $46.26(9.71)$ & $43.95(10.31)$ & 36.88 (9.17) & $F(2,105)=7.33$ & .001 & 1.0 & .001 & .011 \\
\hline \multicolumn{9}{|l|}{ WCST } \\
\hline Failures to maintain sets & $19.07(5.94)$ & $20.33(8.03)$ & $25.28(12.67)$ & $F(2,107)=4.27$ & .016 & 1.0 & .018 & .069 \\
\hline Perseveration score & $18.05(15.20)$ & $17.60(14.32)$ & $24.33(18.84)$ & $F(2,107)=1.77$ & .176 & 1.0 & .346 & .248 \\
\hline TMT-B & $57.47(18.70)$ & $58.82(17.98)$ & 84.26 (37.29) & $F(2,105)=12.81$ & $<.001$ & 1.0 & $<.001$ & $<.001$ \\
\hline
\end{tabular}

Note: Data are presented as mean (standard deviation). Results of neuropsychological domains are presented as standardized $t$-values. Results of the WCST and TMT-B indicate raw scores.

Abbreviations: ARMS=at-risk mental state; FEP=first episode of psychosis; HC=healthy controls; MATRICS=Measurement and Treatment Research to Improve Cognition in Schizophrenia; $\mathrm{MCCB}=$ MATRICS Cognitive Consensus Battery; TMT-B=Trail Making Test, version B; vs. =versus, WCST $=$ Wisconsin Card Sorting Test. 
Table 3

Mean plausibility ratings for all interpretation types for the consecutive sentences $1-3$.

\begin{tabular}{llll}
\hline & HC $(\mathrm{n}=38)$ & ARMS $(\mathrm{n}=44)$ & FEP $(\mathrm{n}=28)$ \\
\hline TRUE 1 & $2.73(1.90)$ & $2.50(1.61)$ & $3.01(2.41)$ \\
TRUE 2 & $3.70(1.59)$ & $3.59(1.53)$ & $3.74(1.86)$ \\
TRUE 3 & $8.69(1.68)$ & $8.35(1.91)$ & $7.74(2.34)$ \\
Lure 1 & $4.52(1.79)$ & $4.32(1.52)$ & $4.59(2.42)$ \\
Lure 2 & $3.85(1.38)$ & $3.73(1.21)$ & $3.96(1.86)$ \\
Lure 3 & $1.13(.95)$ & $1.34(1.14)$ & $2.15(1.93)$ \\
Absurd 1 & $.78(1.03)$ & $.75(.91)$ & $1.39(1.61)$ \\
Absurd 2 & $.52(.88)$ & $.34(3.41)$ & $.93(1.21)$ \\
Absurd 3 & $.32(1.21)$ & $.14(.34)$ & $.62(1.03)$ \\
\hline
\end{tabular}

Note: Data are presented as mean (standard deviation). Data of lure-ratings comprise mean plausibility ratings of emotional and neutral lures.

Abbreviations $\mathrm{ARMS}=$ at risk mental state, $\mathrm{FEP}=$ first episode of psychosis, $\mathrm{HC}=$ healthy controls

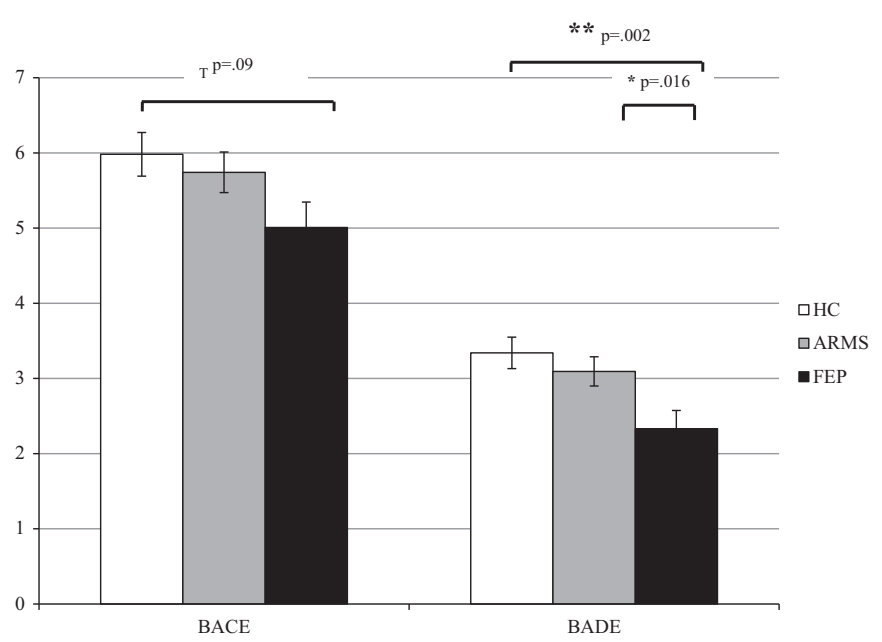

Fig. 1. Patterns of evidence integration. Displayed are estimated marginal means and standard errors. FEP-patients differed significantly from both groups in the integration of disconfirmatory evidence (BADE) and showed a trend towards significance regarding the bias against confirmatory evidence (BACE) compared with the control group. The results of ARMS-patients lay in between the other groups without reaching significant differences. Abbreviations ARMS = at-risk mental state, $\mathrm{BACE}=$ bias against confirmatory evidence, $\mathrm{BADE}=$ bias against disconfirmatory evidence, $\mathrm{FEP}=$ first episode of psychosis.

Bonferroni correction for multiple testing showed that FEP-patients integrated disconfirmatory evidence significantly less than healthy controls $(p=.002,95 \% \mathrm{CI}[.37,1.65])$ and ARMS-patients ( $p=.016,95 \%$ CI $[.14,1.38])$, whereas there was no difference between ARMS-patients and controls $(p=.392,95 \% \mathrm{CI}[-.32, .81])$. There was further a trend towards significance regarding the integration of confirmatory evidence $(F(2,106)=2.51, p=.086$, $\eta_{p}^{2}=.05$ ) (Fig. 1).

\subsection{Secondary analysis}

Correlations in the "all-patients"-group were found with medium effect sizes between BADE and positive symptoms, disorganization, delusional conviction and disruption of life by delusions. Furthermore, results revealed correlations with a medium effect between BADE and delusion conviction in the ARMS-group and between BADE, disruption of life by delusions and negative symptoms in the FEP-group (Table 4). However, none of these correlations survived the Bonferroni correction threshold for multiple testing. To understand a possible role of psychopathology in the initial ratings, we analyzed the positive and negative scores of the PANSS in reference to plausibility ratings after sentence 1 .
There were no significant correlations in the ARMS-group. However, FEP-patients with higher negative symptoms scores presented lower ratings for lure $(r=-.565, p=.003)$ and true sentences $(r=-.510, p=.008)$ (Fig. 2). As depressive symptoms are frequent in ARMS-samples, we analyzed the impact of depression on evidence integration by dividing all patients according to Addington et al. (1993) into those with (Calgary Depression Scale for Schizophrenia score $>6$ ) and without (Calgary Depression Scale for Schizophrenia score $\leq 6$ ) clinical relevant depression. Group comparisons revealed no differences for BADE $(t(70)=-.29$, $p=.78,95 \% \mathrm{CI}[-1.18, .88])$ or BACE $(t(70)=-.42, p=.68,95 \% \mathrm{CI}$ $[-1.35, .88])$. Likewise, the amount of benzodiazepine treatment (diazepam equivalents per day) was not associated with BADE $(\mathrm{r}=.25, p=.28)$ or BACE $(r=.19, p=.40)$.

Correlational analyses between BADE, BACE and neuropsychological measures revealed that only social cognition correlated with BADE in the FEP-group and showed a large effect. In the ARMS-group, correlations between BACE and executive functioning measured by the Wisconsin Card Sorting Test reached a medium effect size. None of these correlations reached significance after Bonferroni corrections. There were no significant correlations in the control group.

\subsection{Tertiary analysis}

ARMS-subgroups were compared regarding their evidence integration performance and no group differences were found (BADE: $\left.F(2,40)=.03, p=.97, \eta_{p}^{2}=.001\right)$; BACE: $F(2,40)=.16, p=.85$, $\left.\eta_{p}^{2}=.01\right)$.

\section{Discussion}

Prior research has found a bias in evidence integration, with a main focus on a biased integration of disconfirmatory evidence, in patients with schizophrenia (e.g. Riccaboni et al., 2012; Veckenstedt et al., 2011; Woodward et al., 2006a) and in healthy controls with high delusion scores (e.g. Buchy et al. 2007; Orenes et al., 2012). The present study investigated BADE in patients with a risk constellation for psychosis and antipsychotic-free patients with a first episode of psychosis aiming at increasing our knowledge of this cognitive bias in the pathogenesis of psychosis.

Supporting these prior findings, our results revealed a bias against disconfirmatory evidence in FEP-patients. Thus, already during the first manifestation of a psychosis, patients present impairment in integrating information which does not support their current hypothesis into the reasoning process. There seem to be differential patterns of evidence integration: Whereas FEP-patients were specifically impaired in adapting their incorrect beliefs according to disconfirmatory information, there was only marginal evidence for a potential bias in the integration of confirmatory evidence. Important to note, this is to our knowledge the first study to investigate patients without antipsychotic treatment. Based on previous findings (Andreou et al., 2015), we considered antipsychotic medication to be a potential influence on BADE performance. Our findings now underline that BADE occurs in acutely psychotic patients before their initial regular antipsychotic treatment. There were further no effects of benzodiazepine intake or depressive symptomatology.

The main interest of this study was to investigate evidence integration in risk constellations of psychosis. The ARMS-group did not differ significantly from the other two groups, but a threestaged image of evidence integration performance was revealed. There are different ways to explain these results. On the one hand, a bias in evidence integration might not occur before a first 
Table 4

Correlations between BADE, BACE, psychopathology and neuropsychology.

\begin{tabular}{|c|c|c|c|c|c|c|}
\hline & \multicolumn{3}{|l|}{ BADE } & \multicolumn{3}{|l|}{ BACE } \\
\hline & ARMS patients & FEP patients & All patients & ARMS patients & FEP patients & All patients \\
\hline ERIraos total score & -.04 & - & - & -.07 & - & - \\
\hline \multicolumn{7}{|l|}{ PANSS } \\
\hline Positive symptoms & -.05 & -.16 & -.27 & -.01 & .22 & .01 \\
\hline Negative symptoms & .08 & -.29 & -.15 & -.13 & .21 & -.001 \\
\hline Excitement & -.05 & -.14 & -.14 & -.06 & .09 & -.06 \\
\hline Emotional distress & .09 & -.19 & -.11 & -.03 & -.03 & -.08 \\
\hline Disorganization & -.20 & -.24 & -.30 & -.12 & -.17 & -.26 \\
\hline \multicolumn{7}{|l|}{ PSYRATS-delusions } \\
\hline Conviction & -.32 & -.08 & -.28 & -.10 & .13 & -.13 \\
\hline Disruption & -.29 & -.46 & -.37 & -.07 & .09 & -.11 \\
\hline SANS & .09 & -.38 & -.17 & -.13 & .13 & -.05 \\
\hline CDSS & .02 & .11 & -.05 & -.06 & .14 & .06 \\
\hline \multicolumn{7}{|l|}{$M C C B$} \\
\hline Processing speed & .17 & .36 & .30 & .03 & .13 & .16 \\
\hline Attention/vigilance & .26 & .15 & .22 & .26 & .32 & .32 \\
\hline Working memory & -.09 & .32 & .15 & -.08 & .08 & .06 \\
\hline Verbal learning & .27 & .07 & .20 & -.14 & .04 & .006 \\
\hline Visual learning & -.04 & .03 & -.01 & -.01 & .12 & .09 \\
\hline Problem solving & .04 & .23 & .16 & .14 & -.02 & .15 \\
\hline Social cognition & .17 & .50 & .33 & -.15 & .15 & .04 \\
\hline \multicolumn{7}{|l|}{ WCST } \\
\hline Failures to maintain sets & -.09 & -.03 & -.09 & .31 & -.23 & .10 \\
\hline Perseveration score & -.06 & -.15 & -.08 & .33 & -.24 & .10 \\
\hline TMT-B & -.27 & -.07 & -.22 & -.10 & -.24 & -.25 \\
\hline
\end{tabular}

Note Negative correlations between BADE and measures of positive symptoms and disorganization (PANSS) and delusional symptoms (PSYRATS) in the entire patient group and partly in the separate groups reached medium effect sizes but did not withstand Bonferroni corrections for multiple testing. Social cognition correlated as only neurocognitive domain with BADE in the all-patients-group and in the FEP-group with a large effect. In the ARMS-group, correlations between BACE and executive functioning measured by WCST reached medium effect size. No correlations reached significance after Bonferroni corrections. Correlations were performed using Pearson product-moment correlation coefficient or Spearman Rank correlation.

Abbreviations ARMS = at-risk mental state, $C D S S=$ Calgary Depression Scale for Schizophrenia, ERIraos=Early Recognition Inventory based on IRAOS, FEP = first episode of psychosis, GPP = PANSS general psychopathology, IRAOS = Interview for the Retrospective Assessment of the Onset of Schizophrenia and Other Psychoses, MCCB = MATRICS Consensus Cognitive Battery, PANSS = Positive and Negative Syndrome Scale, PSYRATS=Psychotic Symptom Rating Scales, TMT-B=Trail Making Test, version B, WCST $=$ Wisconsin Card Sorting Test.

episode of psychosis. This interpretation seems to be rather unlikely since prior studies have found a BADE even in healthy people who report a high degree of delusional ideation (Orenes et al., 2012; Zawadzki et al., 2012). More likely, the heterogeneity of the ARMS-sample might have confounded our findings. Only about $22 \%$ of patients meeting ARMS-criteria experience a transition to psychosis after one year (Fusar-Poli et al., 2012; McGorry et al., 2009; Ruhrmann et al., 2010). The remaining group of patients might experience an improvement of symptoms, develop an affective illness, experience a later transition, or have stable symptoms (Fusar-Poli et al., 2012). The current group comprised patients with only cognitive basic symptoms and/or attenuated psychotic symptoms and/or brief limited intermittent psychotic symptoms and therefore covered a variety of psychosis-prone symptoms. The analysis of the different ARMS-subgroups revealed no group differences, but subgroups were probably too small for sufficient statistical power.

The risk constellation for psychosis is characterized by clinically relevant symptoms that do not meet distinct psychotic criteria but that already produce personal distress and disability (Fusar-Poli et al., 2012). Thus, in contrast to many acutely psychotic patients, ARMS-patients are usually help-seeking and show good illness insight. In this respect, a third possible explanation of the current findings might be that ARMS-patients recognize their reasoning biases in the integration of disconfirmatory evidence and concentrate on counter-regulating their answer behavior. This interpretation could also explain why ARMS-patients rated absurd items with the lowest plausibility and significantly different than the FEP-group. They might have implemented an over-cautious response style. Likewise, Barkus et al. (2010) reported that ARMSpatients feel a higher need to control their own thoughts compared to healthy people with schizotypal characteristics, which was associated with high psychological distress. As delusional conviction grows stronger, ARMS-patients might become less and less able to regard newly incoming information and to adapt their beliefs according to disconfirmatory evidence. In line with this hypothesis, Uchida et al. (2014) found higher self-certainty in ARMS-patients to be associated with stronger delusional conviction.

A bias in the integration of disconfirmatory evidence has been regarded a risk factor for delusions, especially for the maintenance of delusional beliefs (Garety et al., 2001) and correlations between belief flexibility and delusion conviction have been reported many times (Sanford et al., 2014; So et al., 2012; Speechley et al., 2012; Woodward et al., 2006b). Therefore, associations between BADE and delusional measures would have been expected in our sample. However, no associations remained significant after Bonferroni corrections for multiple testing. This might, in line with prior literature (Eifler et al., 2014a; Veckenstedt et al., 2011; Woodward et al., 2008), imply that BADE is an underlying factor of psychosis, not state-dependent but rather present in different stages of the illness, independent of medication and independent of delusions. Nevertheless, the associations which were found between delusional measures and BADE all reached medium effect sizes. Also, group difference of BADE pointed towards an increase in evidence integration impairment from an at-risk mental state to a first psychotic episode. Upcoming studies with larger samples and longitudinal investigations are needed in order to resolve whether these non-significant associations with positive symptoms might have been due to limited power.

Different mechanisms have been suggested to underlie belief inflexibility. The "liberal acceptance" theory (Moritz and 

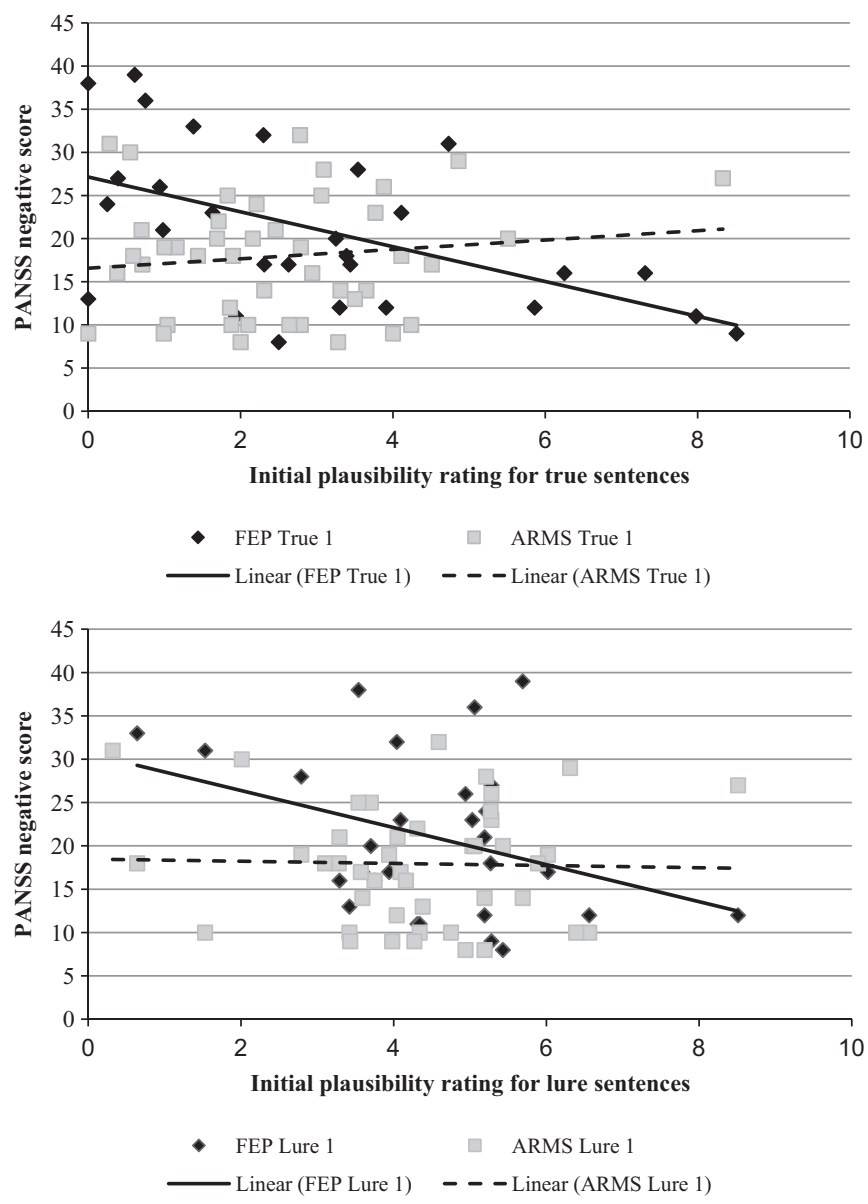

Fig. 2. Correlational analyses showed a significant negative association between negative symptoms scores and the initial plausibility of true $(\mathrm{r}=-.510, \mathrm{p}=.008)$ and lure $(\mathrm{r}=-.565, \mathrm{p}=.003)$ sentences in the FEP group. No correlation could be found in ARMS-patients. Abbreviations ARMS=at-risk mental state, FEP=first episode of psychosis, PANSS=Positive and Negative Syndrome Scale.

Woodward 2004; Moritz et al., 2005) posits that a threshold for acceptance is lowered which under some conditions prompt jumping to conclusions. Consequently, new evidence cannot be integrated to a sufficient amount. Our data support this theory by showing a liberal acceptance of absurd interpretations in the FEPgroup. Liberal acceptance can be explained by the concept of a dysregulated salience attribution (Kapur, 2003). Inappropriate salience can readily trigger high plausibility attributions, even on the basis of little information. Speechley et al. (2012) suggested that patients with psychosis particularly tend to assign a high salience to information which matches their own hypothesis. An impaired integration of disconfirmatory evidence might thus be explained by a "hypersalience of hypothesis-evidence matches" (Speechley et al., 2012). An aberrant salience attribution is closely linked to dopaminergic processes (Andreou et al., 2013). Supporting this, antidopaminergic treatment was found to improve belief flexibility such as evidence integration (Andreou et al., 2015; So et al., 2012). Likewise, higher dopamine levels through the administration of the dopaminergic agonist L-Dopa were associated with less evidence integration (Andreou et al., 2015). Aberrant salience could result from a deficit in correctly updating contextual information or be due to disturbed feedback processing (Andreou et al., 2013). This fits well with neural activation patterns during a probabilistic paradigm of decision making (Rausch et al., $2014,2015)$ and in general with the theory of a deficient error prediction signaling in patients with schizophrenia, leading to an insufficient update of the probability of beliefs in the presence of ambiguous information (Corlett, 2015; Fletcher and Frith, 2009). Again, impaired error prediction signaling has been supposed to arise from abnormal dopamine-modulated processes (Fletcher and Frith, 2009).

Within the scope of the present results, negative symptoms may also be regarded as a potential mechanism. Associations between negative symptoms and BADE are seldom discussed in the literature. Only a few studies reported not finding any association in their data (Garety et al., 2005; Juarez-Ramos et al., 2014; Moritz and Woodward, 2006; Woodward et al., 2007). However, most studies have not even tested for associations between BADE and negative symptoms while concentrating on the positive symptom account as a primary hypothesis. This proceeding in fact involves the risk of seeking only confirmation of hypotheses instead of regarding disconfirmatory evidence. Researchers might thus not be protected from reasoning biases, a topic which has even been currently discussed in the media (Barrett, 2015; The NY Times, 2015 ). The present data gave some indication that higher negative symptoms are associated low initial ratings and with lower integration of disconfirmatory evidence in FEP-patients. Patients with prominent negative symptoms thus might tend to response more conservatively in the beginning of each reasoning process and deficiently consider the information in the course of decision making.

To understand neurocognitive mechanisms of evidence integration we analyzed associations with several neuropsychological domains. As expected, groups differed significantly (Eisenacher et al., submitted for publication), but BADE and BACE did not correlate significantly with specific domains of the MATRICS after corrections for multiple testing. To a large extent, evidence integration seems to be independent of the integrity of neurocognitive functions. This is in accordance with some reports of prior studies (Moritz et al., 2010; Woodward et al., 2007) but opposite findings have also been reported (Buonocore et al., 2015; Eifler et al., 2014a; Riccaboni et al., 2012). In our study, the association between BADE and social cognition reached a large effect in the FEP-group. In line with this, Riccaboni et al. (2012) found that BADE in psychosis patients was not independent of the performance in a theory of mind task. As no results of our correlational analyses withstood corrections for multiple testing, we suggest conducting replication studies to gain better insight in interrelations. An interdependence between different cognitive functions is likely (Fernandez-Duque et al., 2000; Lysaker et al., 2008) and can also be found regarding other metacognitive biases (Eifler et al., 2014a; Garety et al., 2013, Woodward et al., 2009). In future, studies with larger ARMS-samples are needed. Buonocore et al. (2015) found an effect of additional metacognitive training but not neurocognitive treatment alone on BADE, though cognitive measures were intercorrelated before treatment. A better understanding of intercorrelations will improve metacognitive and neurocognitive trainings. Possibly, an integration of these interventions might be valuable.

Some limitations must be considered in the interpretation of our results. We aimed at a cross-sectional investigation of evidence integration in risk constellations of psychosis and not specifically in the pre-psychotic stage. Therefore, we did not report longitudinal data and the current data cannot be extrapolated on transitions to psychosis. Especially regarding correlational analyses, the relatively small sample size might have led to Type II errors. Albeit no significant associations were revealed, confounding effects of pharmacological treatment cannot be excluded, but neither benzodiazepines nor antidepressants were previously linked with a risk for BADE.

To our knowledge, the present study is the first to investigate evidence integration in risk constellations of psychosis and patients with a first exacerbation without antipsychotic treatment. 
Summarized, the study revealed a BADE in FEP-patients and a trend towards a BACE. ARMS-patients did not significantly differ from controls but their performance was intermediate between FEP-patients and the control group. These findings imply that impairment in evidence integration appears on a continuum of psychosis development and may tentatively suggest BADE as a cognitive marker for delusions. However, a lack of knowledge on the relationships between BADE and other psychiatric conditions precludes the conclusion that this cognitive bias serves as specific marker for the illness of psychosis. An early metacognitive training could already be beneficial in the ARMS. An early knowledge and awareness of one's cognitive biases likely induces improvement of evidence integration and belief flexibility and potentially prevents further cognitive distortions which may lead to delusions.

\section{Funding}

M.Z., A.M.-L., and P.K. were funded by the Deutsche Forschungsgesellschaft (DFG, http://www.dfg.de, projects ZI1253/3-1, ZI1253/3-2, KI 576/14-2, ME 1591/6-2). S.E. was supported by a Grant of Heidelberg University (Landesgraduiertenförderungsgesetz), D.M. by the Olympia-Morata Program, and F.S. by the Evangelisches Studienwerk and by the Deutscher Akademischer Austauschdienst (DAAD). The funders had no role in study design, data collection and analysis, decision to publish or preparation of the manuscript.

\section{Conflicts of interest}

S.Ei, F.R., D.M., S., F. R.V., A.B., C.A., S.M., and P.K.: None. S.En. has received travel expenses and consultant fees from AstraZeneca, Bristol-Myers Squibb GmbH \& CoKGaA, Eli-Lilly, Janssen Cilag, Otsuka Pharma, Pfizer Pharma and Servier. A.M.-L. received consultant fees and travel expenses from AstraZeneca, Hoffmann-La Roche, Lundbeck Foundation. Speaker's fees from Pfizer Pharma, Lilly Deutschland, Glaxo SmithKline, Janssen Cilag, Bristol-Myers Squibb, Lundbeck, Servier and AstraZeneca. M.Z. received unrestricted scientific grants of the German Research Foundation (DFG), and Servier; further speaker and travel grants were provided from Pfizer Pharma GmbH, Bristol Myers Squibb Pharmaceuticals, Otsuka, Servier, Lundbeck, Janssen Cilag, Roche, Ferrer and Trommsdorff.

\section{Acknowledgments}

We thank all our participants. We are grateful to the staff of the inpatient clinic who helped support our study appointments.

\section{Appendix A}

Example scenario of the BADE task:

Sentence 1: Simone is tense.

Sentence 2: This day is decisive for her future life.

Sentence 3: Simone will have to receive chemotherapy.

Interpretation 1: Simone is a little nervous because she will have to hold a presentation.

Interpretation 2: Simone will receive the results of her final examinations today.

Interpretation 3: Simone has received a diagnosis of cancer today.

Interpretation 4: Simone takes part in a mars-expedition

\section{References}

Addington, D., Addington, J., Maticka-Tyndale, E., 1993. Assessing depression in schizophrenia: the Calgary depression scale. Br. J. Psychiatry 163 (Suppl 22), $39-44$.

Andreasen, N.C., 1989. The scale for the assessment of negative symptoms: conceptual and theoretical foundations. Br. J. Psychiatry 7, 49-58.

Andreou, C. Schneider, B.C., Braun, V., Kolbeck, K., Gallinat, J., Moritz, S., 2015. Dopamine effects on evidence gathering and integration. J. of Psychiatry and. Neurosci. 40,4

Andreou, C., Moritz, S., Veith, K., Veckenstedt, R., Naber, D., 2013. Dopaminergic modulation of probabilistic reasoning and overconfidence in errors:a doubleblind study. Schizophr. Bull. 40 (3), 558-565.

Ashton, C.H., 2002. The Ashton Manual. 〈http://www.benzo.org.uk/manual/index. htm>, (accesssed 10.02.15)

Barkus, E., Stirling, J., French, P., Morrison, A., Bentall, R., Lewis, S., 2010. Distress and metacognition in psychosis prone individuals: comparing High schizotypy to the at-risk mental state. J. Nerv. Ment. Dis. 198 (2), 99-104.

Barrett, L.F., 2015. Psychology is Not in Crisis. 〈http://www.nytimes.com/2015/09/ 01/opinion/psychology-Is-Not-in-Crisis.HTML?_r=0 (accessed 2109.15).

Buchy, L., Woodward, T.S., Liotti, M., 2007. A cognitive bias against disconfirmatory evidence (BADE) is associated with schizotypy. Schizophr. Res. 90 (1-3), 334-337.

Buonocore, M., Bosia, M., Riccaboni, R., Bechi, M., Spangaro, M., Piantanida, M., Cocchi, F., Guglielmino, C., Bianchi, L., Smeraldi, E., Cavallaro, R., 2015. Combined neurocognitive and metacognitive rehabilitation in schizophrenia: effects on bias against disconfirmatory evidence. Eur. Psychiatry 30 (5), 615-621.

Corlett, P.R., 2015. Answering some phenomenal challenges to the prediction error model of delusions. World Psychiatry 14 (2), 181-183. http://dx.doi.org/ $10.1002 /$ wps.20211.

Eifler, S., Rausch, F., Schirmbeck, F., Veckenstedt, R., Englisch, S., Meyer-Lindenberg, A., Kirsch, P., Zink, M., 2014a. Neurocognitive capabilities modulate the integration of evidence in schizophrenia. Psychiatry Res. 219 (7), 72-78.

Eifler, S., Rausch, F., Schirmbeck, F., Veckenstedt, R., Mier, D., Esslinger, C., Englisch, S., Meyer-Lindenberg, A., Kirsch, P., Zink, M., 2014b. Metamemory in schizophrenia: retrospective confidence ratings interact with neurocognitive deficits. Psychiatry Res. 225 (3), 596-603.

Eisenacher, S., Rausch, F., Ainser, F., Englisch, S., Becker, A., Mier, D., Fenske, S., Meyer-Lindenberg, A., Kirsch, P., Zink, M., 2016. Basic Symptoms and Ultra-High Risk criteria Are accompanied by Neurocognitive impairment in Patients with an "at-Risk Mental State" for Psychosis. Early Interv. Psychiatry. (submitted for publication).

Eisenacher, S., Rausch, F., Ainser, F., Mier, D., Veckenstedt, R., Schirmbeck, F., Lewien, A., Englisch, S., Andreou, C. Moritz, S., Meyer-Lindenberg A., Kirsch, P. Zink, M. 2015. Investigation of metamemory functioning in the at-risk mental state for psychosis. Psychol. Med. 45 (15), 3329-3340.

Esslinger, C., Braun, U., Schirmbeck, F., Santos, A., Meyer-Lindenberg, A., Zink, M., Kirsch, P., 2013. Activation of midbrain and ventral striatal regions implicates salience processing during a modified beads task. PLoS One 8 (3), e58536, doi:58510.51371/journal.pone.0058536.

Fernandez-Duque, D., Baird, J.A., Posner, M.I., 2000. Executive attention and metacognitive regulation. Conscious. Cogn. 9 (2), 288-307.

Fine, C., Gardner, M., Craigie, J., Gold, I., 2007. Hopping, skipping or jumping to conclusions? clarifying the role of the JTC bias in delusions. Cogn. Neuropsychiatry $12(1), 46-77$.

Fletcher, P.C., Frith, C.D., 2009. Perceiving is believing: a Bayesian approach to explaining the positive symptoms of schizophrenia. Nat. Rev. Neurosci. 10 (1) $48-58$.

Fusar-Poli, P., Bonoldi, I., Yung, A.R., Borgwardt, S., Kempton, M.J., Valmaggia, L., Barale, F., Caverzasi, E., McGuire, P., 2012. Predicting psychosis: meta-analysis of transition outcomes in individuals at high clinical risk. Arch. Gen. Psychiatry 69 (3), 220-229.

Fusar-Poli, P., Borgwardt, S., Bechdolf, A., Addington, J., Riecher-Rossler, A., SchultzeLutter, F., Keshavan, M., Wood, S., Ruhrmann, S., Seidman, L.J., Valmaggia, L., Cannon, T., Velthorst, E., de Haan, L., Cornblatt, B., Bonoldi, I., Birchwood, M., McGlashan, T., Carpenter, W., McGorry, P., Klosterkotter, J., McGuire, P., Yung, A. R., 2013. The psychosis high-risk state: a comprehensive state-of-the-art review. JAMA Psychiatry 70 (1), 107-120.

Garety, P.A., Freeman, D., Jolley, S., Dunn, G., Bebbington, P.E., Fowler, D.G., Kuipers, E., Dudley, R., 2005. Reasoning, emotions, and delusional conviction in psychosis. J. Abnorm. Psychol. 114 (3), 373-384.

Garety, P., Joyce, E., Jolley, S., Emsley, R., Waller, H., Kuipers, E., Bebbington, P., Fowler, D., Dunn, G., Freeman, D., 2013. Neuropsychological functioning and jumping to conclusions in delusions. Schizophr. Res. 150 (2-3), 570-574.

Garety, P., Kuipers, E., Fowler, D., Freeman, D., Bebbington, P., 2001. A cognitive model of the positive symptoms of psychosis. Psychol. Med. 31, 189-195.

Haddock, G., McCarron, J., Tarrier, N., Faragher, E.B., 1999. Scales to measure dimensions of hallucinations and delusions: the psychotic symptom rating scales (PSYRATS). Psychol. Med. 29 (4), 879-889.

Häfner, H., Bechdolf, A., Klosterkoetter, J., Maurer, K., 2012. Psychosen-Früherkennung und Frühintervention. Der Praxisleitfaden. Schattauer, Stuttgart, Germany.

Heaton, R.K., Chelune, G.J., Talley, J., Kay, G.G., Curtis, G., 1993. Wisconsin Card Sorting Test. Manual. Psychological Assessment Resources, Odessa, FL

Jablensky, A., 2013. Karl jaspers: psychiatrist, philosopher, humanist. Schizophr. 
Bull. 39 (2), 239-241.

Jones, S.H., Thornicroft, G. Coffey, M., Dunn, G., 1995. A brief mental health outcome scale: reliability and validity of the global assessment of functioning (GAF). Br. J. Psychiatry 16 (5), 654-659.

Jolley, S., Thompson, C., Hurley, J., Butler, L., Bebbington, P., Dunn, G., Freeman, D. Fowler, D., Kuipers, E., Garety, P., 2014. Jumping to the wrong conclusions? An investigation of the mechanisms of reasoning errors in delusions. Psychiatry Res. 219, 275-282.

Juarez-Ramos, V., Rubio, J.L., Delpero, C., Mioni, G., Stablum, F., Gomez-Milan, E. 2014. Jumping to conclusions bias, BADE and feedback sensitivity in schizophrenia and schizotypy. Conscious. Cogn. 26, 133-144.

Kapur, S., 2003. Psychosis as a state of aberrant salience: a framework linking biology, phenomenology, and pharmacology in schizophrenia. Am J Psychiatry $160(1), 13-23$

Kay, S.R., Fiszbein, A., Opler, L.A., 1987. The positive and negative syndrome scale (PANSS) for schizophrenia. Schizophr. Bull. 13 (2), 261-276.

Lehrl, S., 2005. Mehrfachwahl-Wortschatz-Intelligenztest MWT-B, unveränderte Auflage ed.5. Spitta Verlag, Balingen, Germany, p. 5

Lysaker, P.H., Warman, D.M., Dimaggio, G., Procacci, M., LaRocco, V.A., Clark, L.K., Dike, C.A., Nicol'o, G., 2008. Metacognition in schizophrenia: associations with multiple assessments of executive function. J. Nerv. Ment. Dis. 196 (5), 384-389.

McGorry, P.D., Nelson, B., Amminger, G.P., Bechdolf, A., Francey, S.M., Berger, G., Riecher-Rossler, A., Klosterkotter, J., Ruhrmann, S., Schultze-Lutter, F., Nordentoft, M., Hickie, I., McGuire, P., Berk, M., Chen, E.Y., Keshavan, M.S., Yung, A.R. 2009. Intervention in individuals at ultra-high risk for psychosis: a review and future directions. J. Clin. Psychiatry 70, 9.

Moritz, S., Veckenstedt, R., Hottenrott, B., Woodward, T.S., Randjbar, S., Lincoln, T.M. 2010. Different sides of the same coin? intercorrelations of cognitive biases in schizophrenia. Cogn. Neuropsychiatry 15, 406-421.

Moritz, S., Woodward, T.S., 2004. Plausibility judgement in schizophrenic patients: evidence for a liberal acceptance bias. Ger. J. Psychiatry 7, 66-74.

Moritz, S., Woodward, T.S., 2006. A generalized bias against disconfirmatory evidence in schizophrenia. Psychiatry Res. 142 (2), 157-165.

Moritz, S., Woodward, T.S., Whitman, J.C., Cuttler, C., 2005. Confidence in errors as a possible basis for delusions in schizophrenia. J. Nerv. Ment. Dis. 193 (1), 9-16.

National Institute of Mental Health, 1970. Chapter 12 - CGI. Clinical Global Impression. In: Guy, W. (Ed.), EDCEU Assessment Manual for Psychopharmacology. U.S. Department of Health, Education, and Welfare, Rockeville, MD pp. 217-222.

Nuechterlein, K.H., Green, M.F, Kern, R.S., Baade, L.E, Barch, D.M., Cohen, J.D., Essock, S., Fenton, W.S., Frese III, F.J., Gold, J.M., Golberg, T., Heaton, R.K., Keefe, R. S., Kraemer, H., Mesholam-Gately, R., Seidman, E.J., Stover, E., Weinberger, D.R. Young, A., Zalcman, S., Marder, S. R., S., 2008. The MATRICS consensus cognitive battery, part 1: test selection, reliability, and validity. Am. J. Psychiatry 165 (2), 203-213.

Orenes, I., Navarrete, G., Beltrán, D., Santamaría, C., 2012. Schizotypal people stick longer to their first choices. Psychiatry Res. 200 (2-3), 620-628.

Patrick, D.L., Burns, T., Morosini, P., Rothman, M., Gagnon, D.D., Wild, D. Adriaenssen, I., 2009. Reliability, validity and ability to detect change of clinician-rated personal and social performance scale in patients with acute symptoms of schizophrenia. Curr. Med. Res. Opin. 25 (2), 325-338.

Rausch, F., Eifler, S., Esser, A., Esslinger, C., Schirmbeck, F., Meyer-Lindenberg, A. Zink, M., 2013. The early recognition inventory ERIraos detects at risk menta States of psychosis with High sensitivity. Compr. Psychiatry 54 (7), 1068-1076. http://dx.doi.org/10.1016/j.comppsych.2013.04.016 [doi].

Rausch, F., Mier, D., Eifler, S., Esslinger, C., Schilling, C., Schirmbeck, F., Meyer-Lindenberg, A., Kirsch, P., Zink, M., 2014. Reduced activation in ventral striatum and ventral tegmental area during probabilistic decision-making in schizophrenia. Schizophr. Res. 156 (2-3), 143-149. http://dx.doi.org/10.1016/j. schres.2014.04.020 [doi].

Rausch, F., Mier, D., Eifler, S., Fenske, S., Schilling, C., Schirmbeck, F., Englisch, S., Meyer-Lindenberg, A., Kirsch, P., Zink, M., 2015. Reduced activation in ventral striatum during probabilistic decision-making in "at-risk mental state"-patients. J. Psychiatry Neurosci. 40 (3), 163-173.

Reitan, R.M., Wolfson, D., 1995. Category test and trail making test as measures of frontal lobe functions. Clin. Neuropsychol. 9 (1), 50-56.

Riccaboni, R., Fresi, F., Bosia, M., Buonocore, M., Leiba, N., Smeraldi, E., Cavallaro, R. 2012. Patterns of evidence integration in schizophrenia and delusion.
Psychiatry Res. 200 (2-3), 108-114.

Ruhrmann, S., Schultze-Lutter, F. Salokangas, R.K R, Heinimaa, M. Linszen, D., Dingemans, P., Birchwood, M., Patterson, P., Juckel, G., Heinz, A., Morrison, A., Lewis, S., Graf von Revetlow, H., Klosterkotter, J., 2010. Prediction of psychosis in adolescents and young adults at high risk: results from the prospective European prediction of psychosis study. Arch. Gen. Psychiatry 67 (3), 241-251.

Sanford, N., Veckenstedt, R., Moritz, S., Balzan, R.P., Woodward, T.S., 2014. Impaired integration of disambiguating evidence in delusional schizophrenia patients. Psychol. Med., 1-10. http://dx.doi.org/10.1017/S0033291714000397 [doi].

Saß, H., Wittchen, H., Zaudig, M., 2000. Diagnostisches und statistisches Manual psychischer Störungen DSM-IV. Hogrefe, Göttingen, Germany.

Schultze-Lutter, F., Ruhrmann, S., Berning, J., Maier, W., Klosterkotter, J., 2010. Basic symptoms and ultrahigh risk criteria: symptom development in the initial prodromal state. Schizophr. Bull. 36 (1), 182-191.

Schultze-Lutter, F., Ruhrmann, S., Fusar-Poli, P., Bechdolf, A., Schimmelmann, B.G., Klosterkotter, J., 2012. Basic symptoms and the prediction of first-episode psychosis. Curr. Pharm. Des. 18 (4), 351-357.

Sheehan, D.V., Lecrubier, Y., Sheehan, K.H., Amorim, P., Janavs, J., Weiller, E., Hergueta, T., Baker, R., Dunbar, G.C., 1998. The Mini-International Neuropsychiatric interview (M.I.N.I): the development and validation of a structured diagnostic psychiatric interview for DSM-IV and ICD-10. J. Clin. Psychiatry 59 (20), 22-33.

So, S.H., Freeman, D., Dunn, G., Kapur, S., Kuipers, E., Bebbington, P., Fowler, D., Garety, P.A., 2012. Jumping to conclusions, a lack of belief flexibility and delusional conviction in psychosis: a longitudinal investigation of the structure, frequency, and relatedness of reasoning biases. J. Abnorm. Psychol. 121 (1), 129-139.

So, S.H., Garety, P.A., Peters, E.R., Kapur, S., 2010. Do antipsychotics improve reasoning biases? A review. Psychosom Med. 72 (7), 681-693.

Speechley, W.J., Ngan, E., Moritz, S., Woodward, T.S., 2012. Impaired evidence integration and delusions in schizophrenia. journal of experimental. Psychopathology 3 (4), 688-701.

The New York Times, 2015. 〈http://www.nytimes.com/2015/09/08/opinion/how-re liable-are-psychology-studies.html?_r=0 , (accessed 21.09.15).

Uchida, T., Matsumoto, K., Ito, F., Ohmuro, N., Miyakoshi, T., Ueno, T., Matsuoka, H. 2014. Relationship between cognitive insight and attenuated delusional symptoms in individuals with at-risk mental state. Psychiatry Res. 217 (1-2), 20-24. http://dx.doi.org/10.1016/j.psychres.2014.01.003.

van der Gaag, M., Hoffman, T., Remijsen, M., Hijman, R., de Haan, L., van Meijel, B., van Haarten, P.M., Valmaggia, L., de Hert, M., Kuipers, A., Wiersma, D., 2006. The five-factor model of the positive and negative syndrome scale II: a ten-fold cross-validation of a revised model. Schizophr. Res. 85 (1-3), 280-287. http: //dx.doi.org/10.1016/j.schres.2006.03.021 [doi]

Veckenstedt, R., Randjbar, S., Vitzthum, F., Hottenrott, B., Woodward, T.S., Moritz, S. 2011. Incorrigibility, jumping to conclusions, and decision threshold in schizophrenia. Cogn. Neuropsychiatry 16 (2), 174-192.

Woodward, T.S., Buchy, L., Moritz, S., Liotti, M., 2007. A bias against disconfirmatory evidence IS associated with delusion proneness in a nonclinical sample. Schizophr. Bull. 33 (4), 1023-1028.

Woodward, T.S., Mizrahi, R., Menon, M., Christensen, B.K., 2009. Correspondences between theory of mind, jumping to conclusions, neuropsychological measures and the symptoms of schizophrenia. Psychiatry Res. 170 (2-3), 119-123.

Woodward, T.S., Moritz, S., Chen, E.Y.H., 2006a. The contribution of a cognitive bias against disconfirmatory evidence (BADE) to delusions: a study in an Asian sample with first episode schizophrenia spectrum disorders. Schizophr. Res. 83 (2-3), 297-298.

Woodward, T.S., Moritz, S., Cuttler, C., Whitman, J.C., 2006b. The contribution of a cognitive bias against Disconfirmatory evidence (BADE) to delusions in schizophrenia. J. Clin. Exp. Neuropsychol. 28 (4), 605-617.

Woodward, T.S., Moritz, S., Menon, M., Kinge, R., 2008. Belief inflexibility in schizophrenia. Cogn. Neuropsychiatry 13 (3), 267-277.

Yung, A.R., Yuen, H.P., McGorry, P.D., Phillips, L.J., Kelly, D., Dell'Olio, M., Francey, S. M., Cosgrave, E.M., Killackey, E., Stanford, C., Godfrey, K., Buckby, J., 2005. Mapping the onset of psychosis: the comprehensive assessment of at-risk mental states. Aust. N. Z. J. Psychiatry 39 (11-12), 964-971.

Zawadzki, J.A., Woodward, T.S., Sokolowski, H.M., Boon, H.S., Wong, A.H.C., Menon, M., 2012. Cognitive factors associated with subclinical delusional ideation in the general population. Psychiatry Res. 197 (3), 345-349. 